\title{
Astuces, farces et magie
}

Les recettes de divertissements du «Bâtiment des recettes » $\left(\mathrm{XVI}^{\mathrm{e}}-\mathrm{XIX}^{\mathrm{e}}\right.$ siècles)

Tips, tricks and magic

\section{Geneviève Deblock}

\section{Q OpenEdition}

Journals

Édition électronique

URL : https://journals.openedition.org/tc/6731

DOI : $10.4000 /$ tc. 6731

ISSN : 1952-420X

Éditeur

Éditions de l'EHESS

\section{Édition imprimée}

Date de publication : 15 décembre 2012

Pagination : 308-323

ISBN : 978-2-7351-1534-1

ISSN : 0248-6016

\section{Référence électronique}

Geneviève Deblock, "Astuces, farces et magie », Techniques \& Culture [En ligne], 59 | 2012, mis en ligne le 15 décembre 2015, consulté le 29 septembre 2022. URL : http://journals.openedition.org/tc/6731 DOI : https://doi.org/10.4000/tc.6731 


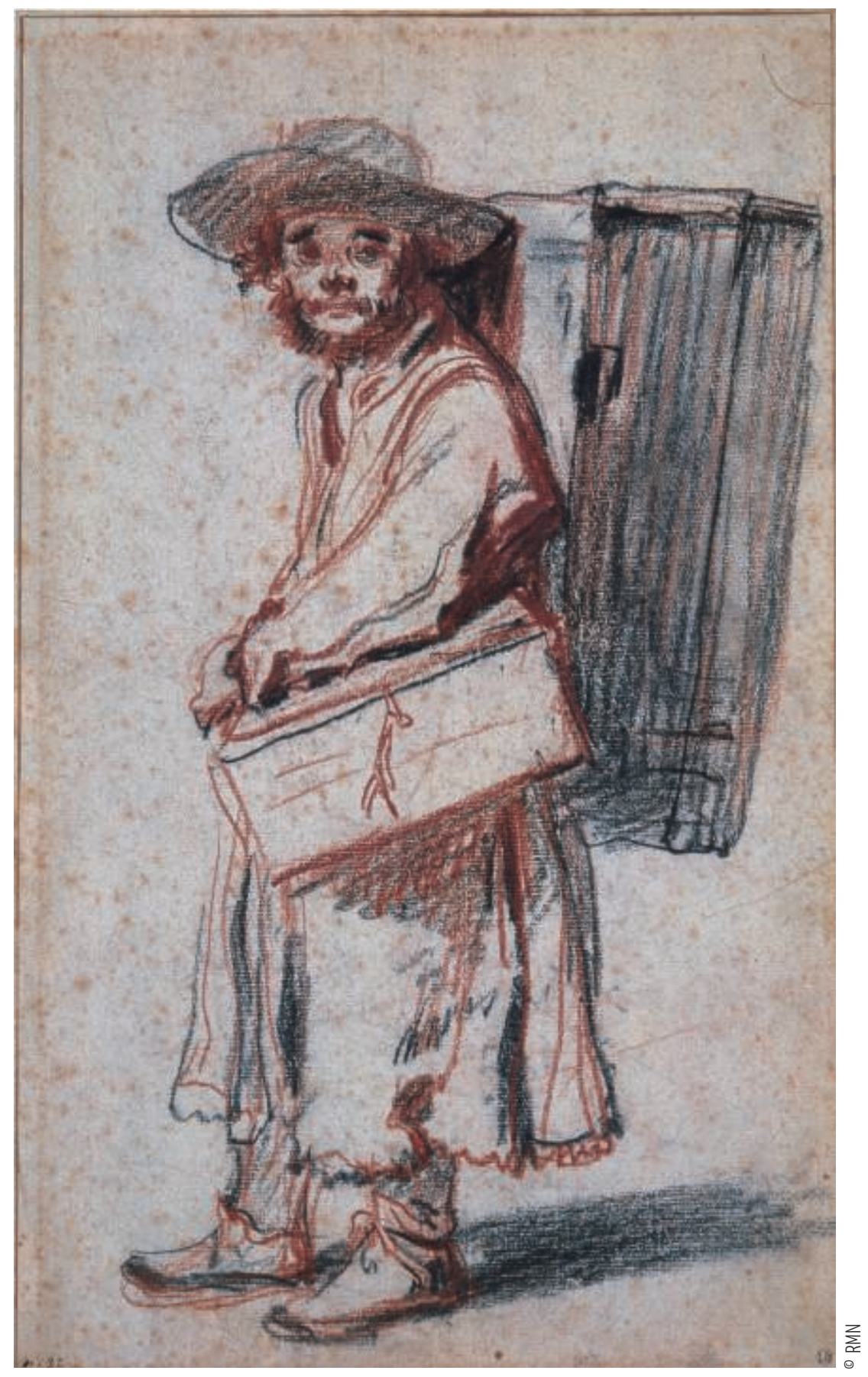




\section{Geneviève Deblock}

\section{ASTUCES, FARCES ET MAGIE}

\section{Les recettes de divertissements

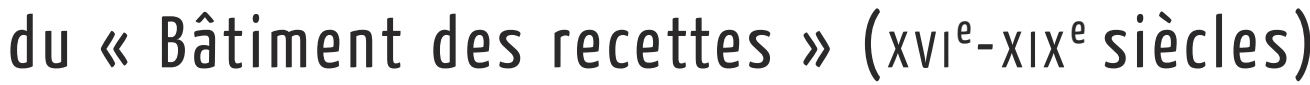

Si l'on a coutume d'observer l'évolution des sciences et des techniques de la période moderne à travers des textes savants et reconnus, le risque est grand d'oublier l'existence d'une autre littérature imprimée, relativement peu étudiée et pourtant très vivante jusqu'au xix siècle, la littérature dite de colportage (Bollème 1971, Morin 1974, Spufford 1984, Mandrou 1985, Fontaine 1993, Delcourt \& Parinet 2000, Bollème \& Andries 2003). L'invention de l'imprimerie au $\mathrm{xv}^{e}$ siècle et la recherche de nouveaux publics et de nouveaux profits sont à l'origine de ces petits livres en langue vulgaire, bon marché et faciles à vendre. Considérés comme sans grande valeur, ils ont largement disparu.

Ce sont des merciers, des colporteurs, « porte-paniers » ou « contre-porteux », comme les nomme Pierre de L'Estoile (1546-1611), qui diffusent à travers la ville divers imprimés, placards, images, canards, chansons, prédictions, almanachs et «fatras » de toutes sortes, auprès d'une clientèle alphabétisée, donc privilégiée, du moins jusqu'aux années 1660 pour la France (Chartier 1983). Ils participent ainsi à l'élargissement d'un public, et d'un marché du livre (Figure 1).

En effet, le livre de colportage, connu au Xvinl ${ }^{e}$ siècle en France sous le nom de Bibliothèque bleue ${ }^{1}$, s'adresse à un public qui ne se reconnaît pas obligatoirement dans la clientèle habituelle des librairies. Il s'en évade en partie dans le panier du mercier ou du colporteur, « qui va vendre ses marchandises par les rues » (Furetière 1690). À Paris, au XVII e siècle, «seul, le petit peuple des colporteurs, autorisés ou non, et des "étalants" ou des libraires improvisés permet au livre et à l'imprimé de se diffuser hors du quartier réservé et d'aller en tout point de la capitale au-devant de la clientèle » (Mellot 2009). Cette activité est purement urbaine jusqu'à la fin du xvII siècle, et d'ailleurs très surveillée, 


\section{Figure 1 \\ Anciens cris de Paris,}

xvie siècle

Image tirée d'un recueil anonyme de gravures sur bois coloriées, et représentant les petits métiers ambulants. « Beaux abc et heures », crie le colporteur à travers les rues.

Il existe des livres d'heures à tous les prix. Cette spécialité parisienne représente souvent l'unique titre des quelques possesseurs de livres au Xvie siècle, et peut servir également d’abécédaire.

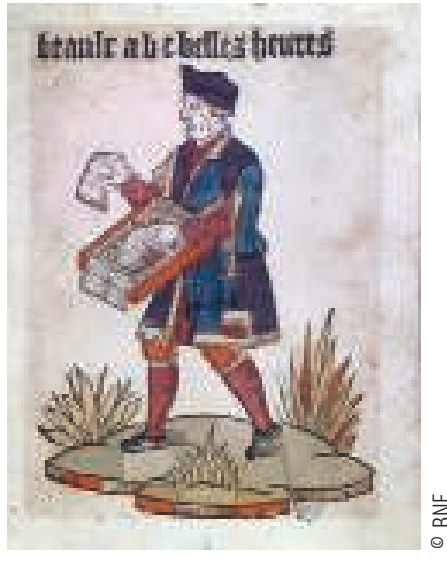

商 car les colporteurs concurrencent les libraires, mais sont aussi des diffuseurs d'imprimés interdits.

$\mathrm{Au} \mathrm{XVI}{ }^{e}$ et au XVII ${ }^{e}$ siècle, le phénomène n'est pas de très grande ampleur. Les livres sont en général assez bien imprimés, et donc probablement encore relativement coûteux. Il s'agit surtout d'anciens titres recyclés. Si la clientèle est principalement urbaine jusqu'à la fin du $\mathrm{XVII}^{\mathrm{e}}$ siècle, et l'on pourrait dire parisienne, elle évolue ensuite. Paris en effet, bénéficie au milieu du XVII siècle d'un bon réseau scolaire, et représente alors près de la moitié du public français alphabétisé ${ }^{2}$. Puis, avec la montée de l'alphabétisation, le colportage se spécialise et se systématise dès la fin du XVII ${ }^{\mathrm{e}}$ siècle. Aux XVIII ${ }^{\mathrm{e}}$ et XIX ${ }^{\mathrm{e}}$ siècles, les tirages des éditions de la Bibliothèque bleue deviennent de plus en plus importants. De véritables réseaux de colporteurs et de « libraires forains » (Sauvy 1983, Mellot 1989) sillonnent les villes mais aussi les campagnes, pour vendre un nombre de plus en plus important d'ouvrages ${ }^{3}$.

Il ne faudrait donc pas sous-estimer le rôle de cette littérature, même si la qualité des éditions se dégrade au cours des siècles, et si elle est de plus en plus mal considérée par l'administration et par les élites. Elle compte quelques ouvrages techniques, ou plus justement pratiques, tels les livrets de secrets ou livrets de recettes ${ }^{4}$. Consultés par un public certainement très divers, ces recueils pratiques ont la particularité de faire de leurs lecteurs des expérimentateurs. L'un d'eux, le Bâtiment des recettes (Deblock à paraître), est la traduction d'une compilation anonyme intitulée Dificio di ricette, qui remporte un vif succès à Venise dans les années 1524-1560 (Figure 2).

Cette traduction française paraît en 1539 , avec divers ajouts de recettes médicales qui l'orientent vers un public plus large et plus diversifié. Le Bâtiment des recettes est ensuite réédité régulièrement jusqu'au $\mathrm{XIX}^{\mathrm{e}}$ siècle ${ }^{5}$. Il est traduit du français en néerlandais en 1546. En 1709, à Rouen, il fait partie d'une liste de vingt-neuf livres réprouvés pour toujours, dont une majorité d'ouvrages religieux et quelques livres profanes. On peut penser que les quatre livres de secrets, d'astrologie et de magie qui font partie de cette liste véhiculent des croyances et des savoirs traditionnels alors encore très ancrés dans la société, mais qui heurtent les idées de progrès du Bureau de la Librairie. Cela ne change toutefois pas grand-chose au parcours éditorial du Bâtiment des recettes, qui se poursuit jusqu'aux premières décennies du xix $x^{e}$ siècle.

Les recettes proposées dans ce petit recueil pratique composé au début du Xvle siècle se rapportent à la vie quotidienne. Les activités domestiques y sont présentes avec une série de recettes de produits de luxe comme les confitures ou les parfums, mais également la conservation des aliments, la chasse, la pêche, l'entretien d'une résidence campagnarde, la fabrication et la conservation du vin, la manière de détacher un tissu ou d'en raviver la couleur, ou bien de raccommoder un plat.

Lartisanat est également présent, avec des recettes de peausserie, de teinture, de fabrication de couleurs, d'encre à écrire, d'encre à imprimer et même d'encres sympathiques. 
La santé et l'entretien du corps représentent le plus grand nombre de recettes : soins de beauté, médecine domestique, dont un certain nombre de secrets expressément pour les fermmes, de recettes contre les maux de dents ou pour se débarrasser de la vermine.

Et parmi ces multiples préoccupations pratiques, un pan important de l'ouvrage concerne divertissements, ironie, bons tours et expériences extraordinaires. Leur présence paraît au premier abord incongrue. C'est pourtant la marque des livrets de charlatans et bateleurs. Ils vendent leur littérature et leurs mixtures dans les rues de Venise et sur la " piazza », centre d'une culture hétéroclite. Le spectacle et le rire sont leur fonds de commerce; l'ordre établi n'est pas leur principale préoccupation (Eamon 1994). Cette dimension ludique et provocatrice du recueil a son importance, car elle abolit certaines frontières, et affranchit le lecteur des limites du quotidien. « Le sérieux est officiel, autoritaire, il s'associe à la violence, aux interdits, aux restrictions, dit Mikhail Bakhtine, contrairement au rire, qui n'impose aucun interdit, aucune restriction » (Bakhtine 1970).

Au xvl' siècle, le Bâtiment des recettes s'adresse, comme le Dificio di ricette, à un public urbain socialement privilégié puisqu'alphabétisé, masculin et aussi féminin (Eamon 2011). Il s’adresse également aux artisans, à ceux que Robert Halleux nomme les « hommes de la pratique » (Halleux 2009), qui ont également accès à l'écrit, officiers, marchands, architectes, ingénieurs, artistes ${ }^{6}$, et nous ajouterions saltimbanques, ou à quelques gentilshommes campagnards, « hobereau, riche laboureur ou maître d'école », tels que le sire de Gouberville ou le riche laboureur Robin Chevet (Martin 1975). Frédéric Barbier parle d'une «nouvelle frange inférieure, alphabétisée, des possédants » (Barbier 2012). Pour

l'Italie, William Eamon parle d'une classe moyenne grandissante, « plus intéressée par des préoccupations matérielles que spirituelles ou purement intellectuelles », et Pamela H. Smith met l'accent sur la croissance d'une culture urbaine et la montée d'une classe moyenne à forte mobilité sociale ne bénéficiant plus de la transmission familiale d'un savoir technique, et désireuse de trouver des conseils de tous ordres (Smith 2011) (Figure 3).

Un exemplaire du Bâtiment des recettes, édité par les frères de Marnef à Poitiers en 1540, est consultable en ligne. C'est à partir de celui-ci que l'on explorera les « plaisir[s] et joyeusetés » qui sont proposés au fil des pages ${ }^{7}$.

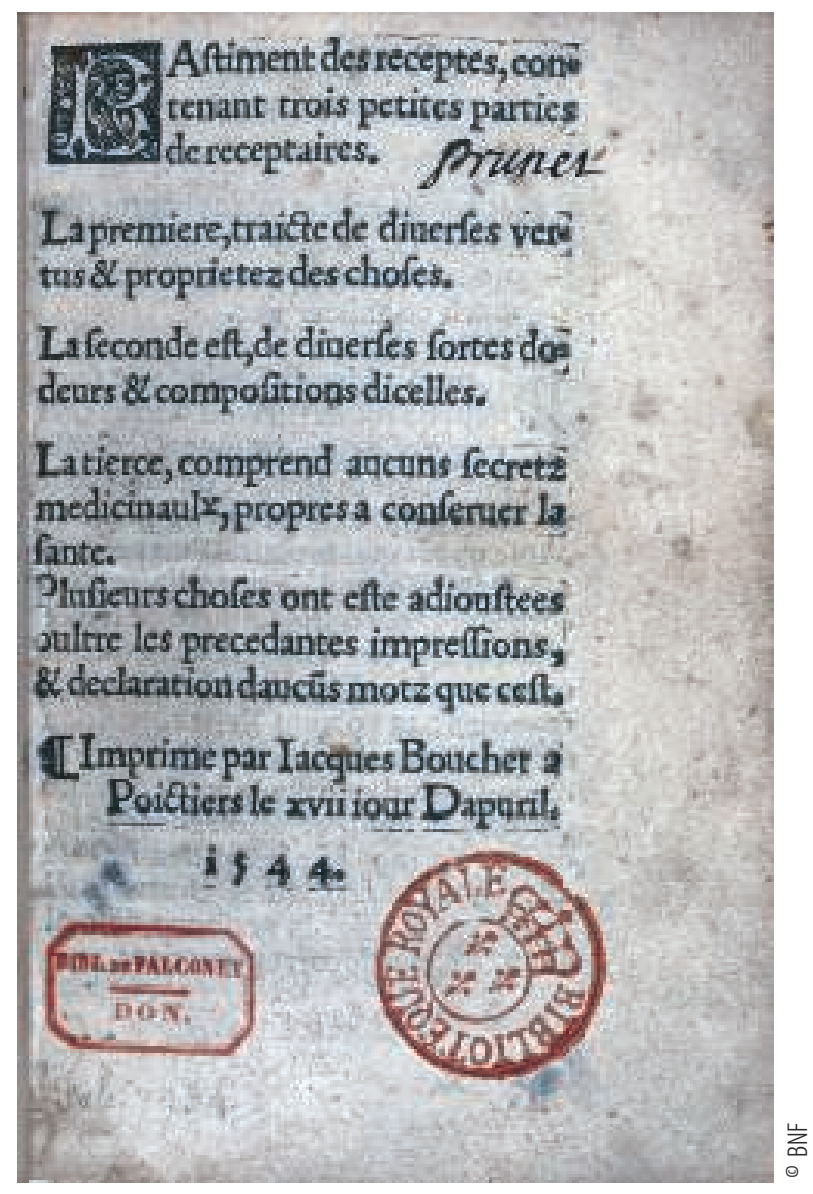

Figure 3

L'édition du Bâtiment des recettes de Jacques Bouchet (1544) est déjà la septième depuis la première traduction française connue (1539). L'annonce plus ou moins vraie de nouveautés est toujours le moteur de la vente (Gallica). « C'est vieux comme le monde, ça, la nouveauté » (Jacques Prévert, Les enfants du Paradis).

\section{Recepte[s] pour faire choses à plaisir \& par joyeuseté}

Les recettes destinées à distraire le lecteur sont dispersées dans l'ouvrage, à l'exception d'une série intitulée "Pour faire choses à plaisir, \& par joyeuseté » (Figure 4). Cet ensemble de treize secrets compte huit pages qui devaient être à l'origine vendues séparément dans les rues de Venise. Il est introduit par ces quelques lignes: 
« Ayant cy dessus escript plusieurs receptes de diverses vertuz, lesquelles sont pour te donner grande utilité, maintenant par manière de passetemps (Lecteur discret) je te vueil escripre aultre[s] petites choses plaisantes, desquelles pourras prendre plaisir \& récréation ».

La lecture de ces treize recettes permet d'identifier le genre de secrets non utiles, mais divertissants que représentent ces passe-temps offerts aux lecteurs : tous emploient des ingrédients faciles à se procurer, et semblent simples à exécuter. Ils enseignent des astuces, des tours, des farces pour s'amuser en société, dont deux expériences fantasmagoriques propres à créer l'effroi.

Le titre de la première recette semble à première vue n'être qu'un jeu d'illusion, une plaisanterie, « Pour faire que ung chien ou cheval te semblera estre tout verd » (f. $42 \mathrm{v}^{\circ}$ ). Il s'agit cependant d'une simple recette de teinture verte obtenue à partir de la distillation de câpres. « L'eau de câpres » est connue. Elle est mentionnée sans autre explication pour teindre des cheveux en vert, dans L'Agriculture et maison rustique de Charles Estienne et Jean Liebaud en 1564, au chapitre XXII intitulé « De la façon de distiller des eaux pour les fards ».

Une série de quatre recettes expliquent ensuite comment jouer avec l'eau et le feu en fabriquant des chandelles qui brûleront dans l'eau, dans la neige ou la glace, ou qui ne s'éteindront jamais, chandelles extraordinaires, mais de fabrication simple : « Pour faire ung cierge ou flambeau de neige que tu pourras allumer \& faire ardoir » (f. $42 \mathrm{v}^{\circ}$ ), «Pour faire que une chandelle puisse ardoir soubz l'eau » (f. $43 \mathrm{r}^{\circ}$ ), «Pour faire une chandelle qui ne s'esteindra jamais au souffler » (f. $43 \mathrm{v}^{\circ}$ ), «Pour faire ung cierge de

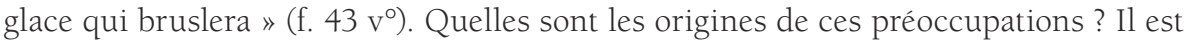
intéressant, pour s'en faire une idée, de se reporter à un autre ouvrage italien publié quelques années plus tard, en 1558, La Magie naturelle ou les Secrets et Miracles de la Nature, du philosophe et alchimiste napolitain Giambattista Della Porta (1535-1615), qui est en somme une encyclopédie des savoirs de l'époque. L'auteur de cet ouvrage de vulgarisation scientifique (Della Porta 1591), traduit et réédité durant deux siècles dans toute l'Europe, présente le fruit de ses lectures et de ses recherches personnelles. À travers des expériences empiriques, il décrit et commente les secrets de la nature, avec un goût particulier pour l'extraordinaire, le merveilleux et le miraculeux, c'est-à-dire l'inexplicable. Il donne l'origine des expériences qu'il présente, raconte et commente. Elles sont souvent tirées des textes anciens. Au livre II, chapitre X, intitulé « Diverses compositions de feux $»^{9}$, il évoque les utilisations possibles de ces chandelles : « des torches que le vent ne peut esteindre », pour guider une armée en marche la nuit contre vent, ou bien une « chandelle de neige ou de glace comme sont les enfans coustumiers de faire ${ }^{10}$, c'est-à-dire un simple jeu d'enfants habituel en hiver.

Suivent deux tours d'illusionnisme : "Pour faire saulter ung anneau par la maison sans que nul le touche » (f. $44 \mathrm{r}^{\circ}$ ), avec l'artifice du salpêtre, du soufre vif et du mercure, et « Pour scavoir cuyre un ouf sans feu » (f. 44 v ), avec de la chaux vive.

Puis viennent deux plaisanteries que l'on peut, à notre époque, trouver de mauvais goût, « Pour faire que une chair cuitte semblera encore estre crue » (f. $44 v^{\circ}$ ) et « Pour faire que une chair cuitte semblera estre toute chargée de vers $»\left(\mathrm{f} .44 \mathrm{v}^{\circ}\right.$ ). Ces farces, certainement destinées à rire à l'occasion de banquets, ne sont pas sans rappeler les nopces de l'enfant de Michel Dael, en la paroisse d'Halluin, que relate Robert Mandrou (Mandrou 1974), et qui se terminent devant la justice : « un certain Guillebert Dumortier, une fois les convives assis se serait ingéré de vouloir faire ung présent à la compagnie avec quelque peu de vau mis sur deux plats dans lesquelles [sic] il avait enclos deux grenouilles... lesquelles à l'ouverture d'iceux plats, ont sauté sur la table et les viandes, ce qui aurait causé ung tumulte et que le tout aurait 

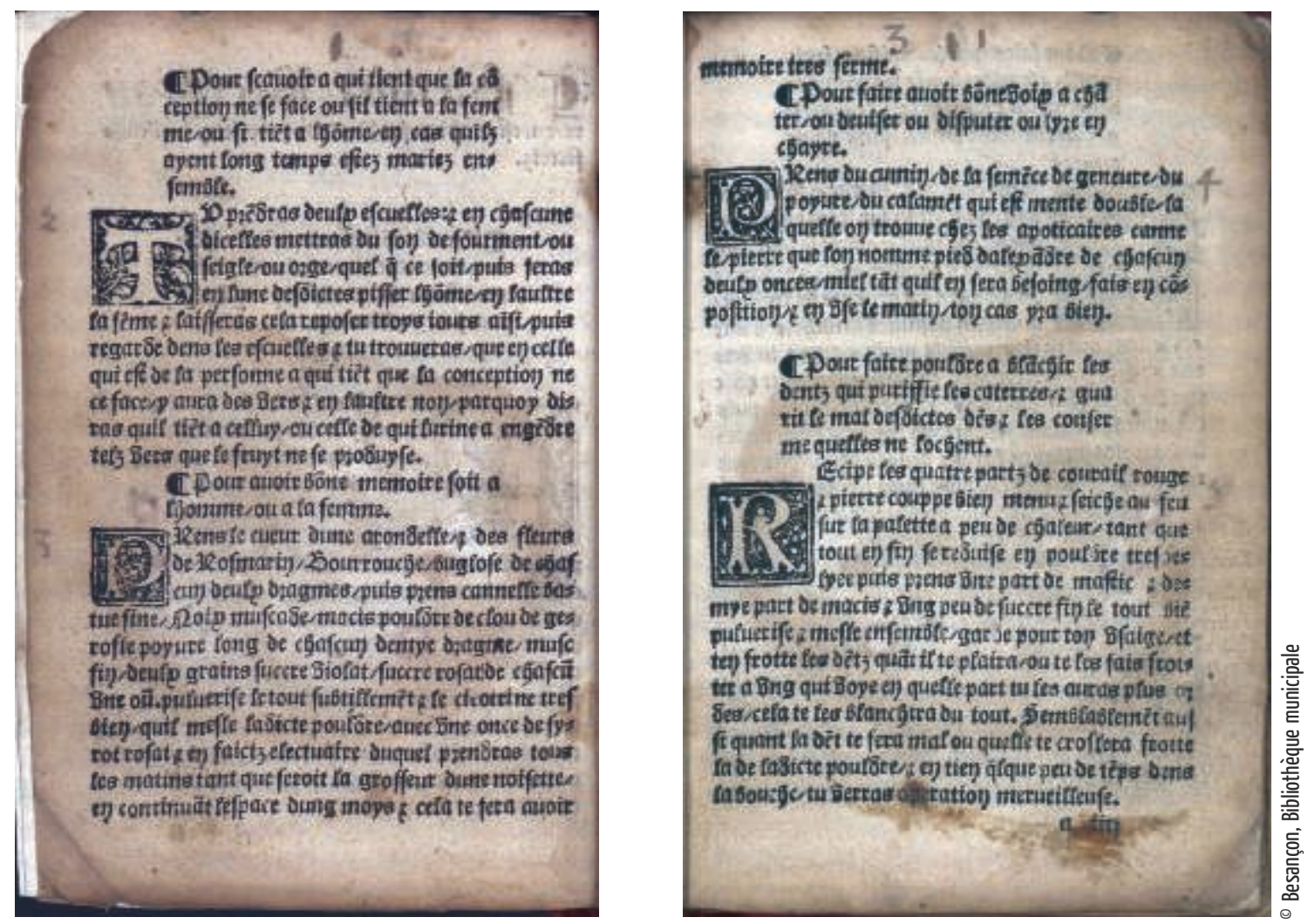

Figure 4

Bâtiment des recettes, Lyon, Olivier Arnoullet, vers 1550, cote BM d'étude : 268699 réserve, pages d2v et d3r.

Le petit texte introductif des « Recettes pour faire choses à plaisir \& par joyeuseté » est noyé au milieu des autres recettes, sans effort de mise en page.

esté culbuté, ce qu’aurait dépleu fort audict remontrant ». Le festin chez Trimalcion, dans le Satyricon de Pétrone, n'est pas très éloigné de ces sortes de mises en scène. Une troisième recette que l'on pourrait également qualifier de « grosse farce », « Pour faire que quelqung ne puisse reposer la nuyct » $\left(\mathrm{f} .45 \mathrm{v}^{\circ}\right)$, consiste à frotter la chemise ou les draps de la personne d'« alun de plume », ou poudre d'alun décomposé, ce qui occasionne de grandes démangeaisons et oblige la personne à se défaire de ses vêtements ou à sortir de son lit.

Deux autres recettes que l'on retrouve également plus tard dans la Magie naturelle de Giambattista Della Porta ${ }^{11}$, utilisent de nouveau des chandelles, mais ici pour créer dans le noir des illusions d'optique terrifiantes: "Pour faire sembler que en une chambre y ayt plusieurs grappes de raisin » (f. $45 \mathrm{r}^{\circ}$ ), «Pour faire sembler que en une chambre y ayt une chasse de bestes saulvaiges » (f. $45 v^{\circ}$ ). On suit dans les écrits de Giambattista Della Porta le déroulement de ses expériences tirées des écrits d'Anaxilas et de Pline, et l'on découvre d'abord la manière de créer des illusions d'optique à partir de l'huile contenue dans une lampe à huile en verre, en opérant surtout dans un environnement obscur. Les premières recettes qu'il propose permettent de créer diverses lumières ambiantes : une lampe en verre vert emplie d'une 
Figure 5

Une troupe de charlatans devant un public de curieux, goguenards, sceptiques, nä̈fs (Rembrandt. Dessin à la plume et à l'encre brune, vers 16371638. Berlin, Kupferstichkabinett).

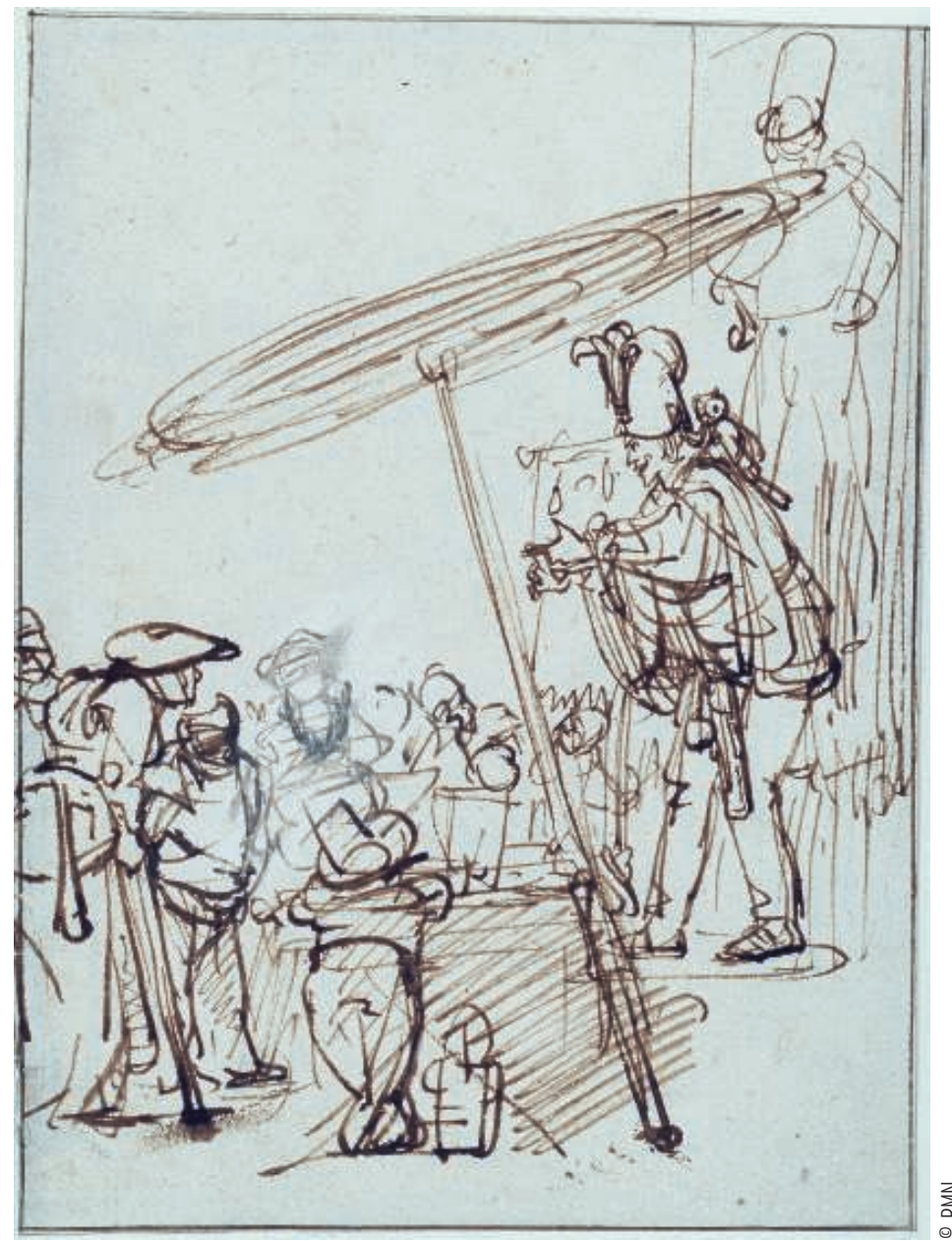

huile mêlée de vert-de-gris « fera apparoir verd tout ce qui sera en la chambre, voir les faces mêsmes des regardans ». Mais elles se combinent trois pages plus loin avec l'utilisation de différents ingrédients comme têtes de chevaux, ou bien grappes de raisins, qui font alors entrer dans la recette une dimension surnaturelle. Par contre, dans le Bâtiment des recettes, ce sont immédiatement ces dernières recettes, les plus terrifiantes mais aussi les plus attractives commercialement, qui sont proposées.

La série se termine, comme elle a commencé, par une plaisanterie, « Pour faire veinr [sic, venir] le poil sur ung bras ou aultre partie du corps qu'il te plaira » (f. $46 \mathrm{r}^{\circ}$ ). Il suffit, selon la recette, d'enduire le bras d'une mixture composée d'huile commune et de poudre de mouches à miel brûlées, « mais garde toy d'en mettre aultre part », ajoute l'auteur, « car le poil y viendroit aussi bien ». Cette moquerie en guise de conclusion exprime bien l'ambiguité du contenu de certaines recettes. En effet, ce secret est une répétition de la recette « Pour faire venir les cheveulx, \& la barbe » (f. $\left.11 \mathrm{r}^{\circ}\right)$. Celle-ci conseille « frotte par plusieurs fois le lieu où vouldras avoir poil : \& tu verras merveille », mais elle n'est pas mentionnée comme une farce au détriment du lecteur naif. Elle est également reprise par Giambattista Della Porta dans la Magie naturelle, Livre II, au chapitre XV, f. 123 v, intitulé « Si vous voulez que le poil naisse avant le temps, où il ajoute il vous naistra du poil mesme à la palme de la main ».

Ces « Recepte pour faire choses à plaisir \& par joyeuseté », sont très diverses. Elles font se côtoyer des jeux, des tours de bateleurs, des plaisanteries, des supercheries, avec des pratiques de magie, un « art qui produit des effets merveilleux par des causes occultes $»^{12}$.

\section{Les autres recettes du Bâtiment des recettes. Une technique au visage du « fripon divin »}

\section{D'autres recettes divertissantes du Bâtiment des} recettes sont dispersées dans le premier réceptaire, où de nouvelles supercheries peuvent se cacher.

La recette « Pour faire brusler ung mouchoir, $\&$ après qu'il sera bruslé, il n'y perra aulcunement, $\&$ demourera encor en son entier, sans avoir eu aucun dommaige » (f. 7 ro) en l'ayant trempé au préalable dans de l'eau-de-vie, et la recette "Pour mettre ung œuf de géline dans une fiolle, ou bocal de verre, qui aye le col estroit » (f. $8 \mathrm{r}^{\circ}$ ) en le ramollissant avec du vinaigre, sont deux tours de saltimbanques. Mais, la recette intitulée « Pour veoir les estoilles en plain midy, ou à quelle heure du jour qu'il te plaira » (f. 6 ro) est-elle une variante incomplète d'une expérience ancienne que Giambattista Della Porta reprend plus tard, toujours dans La Magie naturelle ${ }^{13}$, où il explique que l'expérience n'est pas possible? Ou bien est-ce 
encore, en forme de plaisanterie, une utilisation de l'expression familière au XviI ${ }^{e}$ siècle, « faire voir les étoiles en plein midi à quelqu'un » (Oudin 1971), c'est-à-dire « l'éblouir en lui donnant un grand coup » ou bien l» e tromper ou lui en faire accroire ${ }^{14}$. (Figure 5)

Enfin, une recette apprend au lecteur un tour aux conséquences plus graves, car il donne l'occasion de créer une frayeur collective pour ensuite commettre de petits larcins en toute impunité : « Pour faire une chandelle, laquelle mise sur la table, bien tost après s'esteindra $\&$ fera peur à la compaignie, $\&$ adonc pourras faire sur ladicte table, ce que tu vouldras » $(\mathrm{f} .7 \mathrm{r}$ ). La traduction française est édulcorée. Le titre italien est plus clair et atténue la gravité du larcin par une variante plaisante. Il dit exactement « A far una candela in taovola si smoecera con gran strepita \& fara paura a donne $\&$ ad altri \& poterai far il tuo disegno overo robar

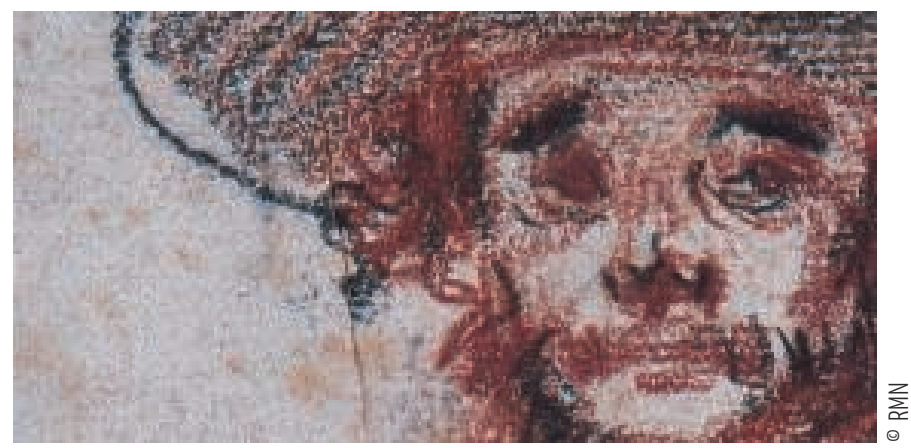
le vivande overo basar la tua inamorata ». Le titre de cette recette sera encore édulcoré, pour devenir très pudiquement au XviIl ${ }^{\mathrm{e}}$ siècle « Pour faire une chandelle, laquelle mise sur la table bien-tôt s'éteindra ». C'est probablement de l'autocensure par crainte d'une nouvelle interdiction d'éditer. (Figure 6)

La technique a plusieurs visages. La " Métis », la « raison rusée » dont parle François Sigaut $(2002,2010)$, a bien dans ces recettes l'image du « trickster » ou «fripon divin ». Si l'on reprend la définition que donne Marcel Mauss de la technique, « un acte traditionnel efficace » (Mauss 1934, 2008), on découvre ici des variantes autour de celle-ci, efficaces mais inutiles, inefficaces, ou bien des recettes aux effets paradoxaux et non traditionnels (Sigaut 2003). Elles peuvent être qualifiées de « ruse et niaiserie, malice et innocence », farce et obscénité d'un Till l'Espiègle que l'on retrouve au XVII siècle dans la littérature de colportage.

\section{Second recueil, le Plaisant jardin : préoccupations semblables}

Les tours et amusements sont encore plus nombreux dans un second recueil également d'origine italienne, le Plaisant jardin des recettes, ajouté au Bâtiment des recettes vers 1550. Ils sont annoncés dans deux petits poèmes publicitaires d'introduction, comme une partie importante du recueil. En effet, est-il écrit avec malice, ajoutés aux recettes médicinales, ils pourront " guarir tant de pieds, mains, que testes $»^{15}$. Leurs vertus de convivialité sont vantées, avec promesse à l'acheteur d'éblouir son auditoire :

Amy Lecteur si tu veulx estre en bruyt

En tout honneur, ou bon êsprit s'applicq[ue],

Porte avec toy ce livret cy traduyct

D’antique langue \& usaige Italique

En pur françoys : lequel met la practicq[ue]

De bien guarir plusieurs maulx survenantz:

\section{Figure 6}

Une étude de colporteur pauvrement vêtu, avec sa boite en bandoulière et sa lourde hotte sur le dos [détail de l'illustration en ouverture] (JeanAntoine Watteau, 1684-1721. Dessin, pierre noire et sanguine. Bayonne, musée Bonnat). 
Pareillement à faire plusieurs tours

De bon esprit, que gens grossiers et lourdz

Ne scauroyent faire s'ilz fussent ilz mal ans.

Tu as icy pour t'amuser

Ce petit livre de Receptes

D'esquelles tu pourras user

Soit en jours ouvriers ou aux festes

En batissant choses honnestes

Pour resjouir tout noble cour

Et guarir tant de pieds, mains, que testes.

Qui te fera bruyt $E$ honneur. ${ }^{16}$

Si ce plaisir annoncé avec vigueur a de quoi étonner le lecteur contemporain, il est cependant bien réel au xvi siècle. On connaît d'autres textes aussi enthousiastes, le mot n'est pas trop fort. William Eamon (2011) mentionne par exemple la dédicace rédigée par le poète Dionigi Atanagi pour son ami Leonardo Fioraventi lors de la sortie de son ouvrage Capricci medicinali, à Venise, en 1564. Celui-ci dit renoncer aux festivités du carnaval et choisir plutôt de lire « ce précieux joyau ». Il loue son style d'écriture, simple, clair, dont le but est d'être compris par le commun des mortels. Ce n'est donc pas uniquement le contenu purement utilitaire qui enthousiasme les lecteurs humanistes du xvl siècle, mais également, au-delà, ce que représente pour la société la simple existence d'un tel ouvrage, en langue vernaculaire, jusqu'alors inaccessible.

Les recettes offrant « grand plaisir \& joyeuseté » peuvent être regroupées selon les genres que l'on a rencontrés précédemment:

Il y a des facéties d'un goût douteux, ou des farces plus ou moins agréables : « Pour peter \& esternuer tout ensemble » (p. 113), ou bien « Pour faire assembler toutes les puces d'une maison en quelque lieu » (p. 115).

Il y a des recettes que l'on pourrait qualifier de culinaires, des farces autour de la nourriture comme on en a vu précédemment : « Pour faire qu’un poullet semblera rosty au plat, s'enfuira quand on le voudra toucher (p. 111) ; « Pour garder le pot de bouillir » (p. 123) ; «A faire que la chair qui est au pot ne cuise point » (p. 123). Il y a également, semble-t-il, des stratagèmes pour mystifier le client, comme « Pour faire reprendre la chair de bœuf couppée en pièces » (p. 123).

Et puis il y a des tours de saltimbanque : « Pour porter feu en la main sans se brusler » (p. 114) ; «A faire monter un ouf jusqu'au bout d'une lance » (p. 115) ; « Pour avoir du feu au soleil » (p. 116); « Pour faire qu'il semble qu'un denier boyve plein un verre d'eau $\gg($ p. 117), etc.

Il y a aussi des recettes extraordinaires utilisant des pratiques occultes, comme les secrets pour créer dans le noir les illusions d'optique terrifiantes que l'on a déjà rencontrées : « Pour faire sembler que les gens ayent teste d'asne » (p. 118) prescrit d'ajouter une cervelle d'âne à l'huile de lampe.

D'autres recettes font appel au fantastique. « Pour faire revenir la veue à un poulet auquel vous avez crevé les yeux » (p. 111), ne se trouve pas dans la partie médicale mais dans la partie générale. Elle concerne ici un poulet et non un homme. Elle consiste à lier sur les yeux du poulet aveuglé une plante nommée « en François piloselle, piluette ou barbette ». Cette plante serait destinée à lui redonner la vue. Il n'est judicieusement pas proposé de tenter cette guérison sur un être humain, mais sur un animal. 
D'autres recettes, comme "A oster la fièvre quarte $»^{17}$ (p. 123), prescrivent des pratiques rituelles occultes. Elles ne se trouvent pas parmi les remèdes, où l'on penserait les voir figurer, mais dans le réceptaire général, ce qui les désigne comme autre chose que des remèdes.

Il est difficile d'identifier les pratiques magiques, car elles peuvent faire appel à des notions ou à des langages dont nous avons perdu les clés. Pamela H. Smith (Smith 2011) explique les significations symboliques, pour les lecteurs de l'époque, de certaines couleurs ou de certains « ingrédients ». Cependant, dès le xvI siècle, une ambiguité est introduite sur l'intention de certaines recettes, souvent grâce au côté ludique du recueil.

Il y a d'ailleurs une logique à présenter sur le même plan les recettes extraordinaires et spectaculaires, que celles-ci relèvent du surnaturel ou bien d'une supercherie ou d'un spectacle. En effet, elles ont toutes un caractère paradoxal (Sigaut 2002, 2010). Les unes sont considérées comme des secrets de nature, les autres relèvent d'un truquage ou d'une facétie. Le compilateur peut ainsi jouer de l'ambiguïté.

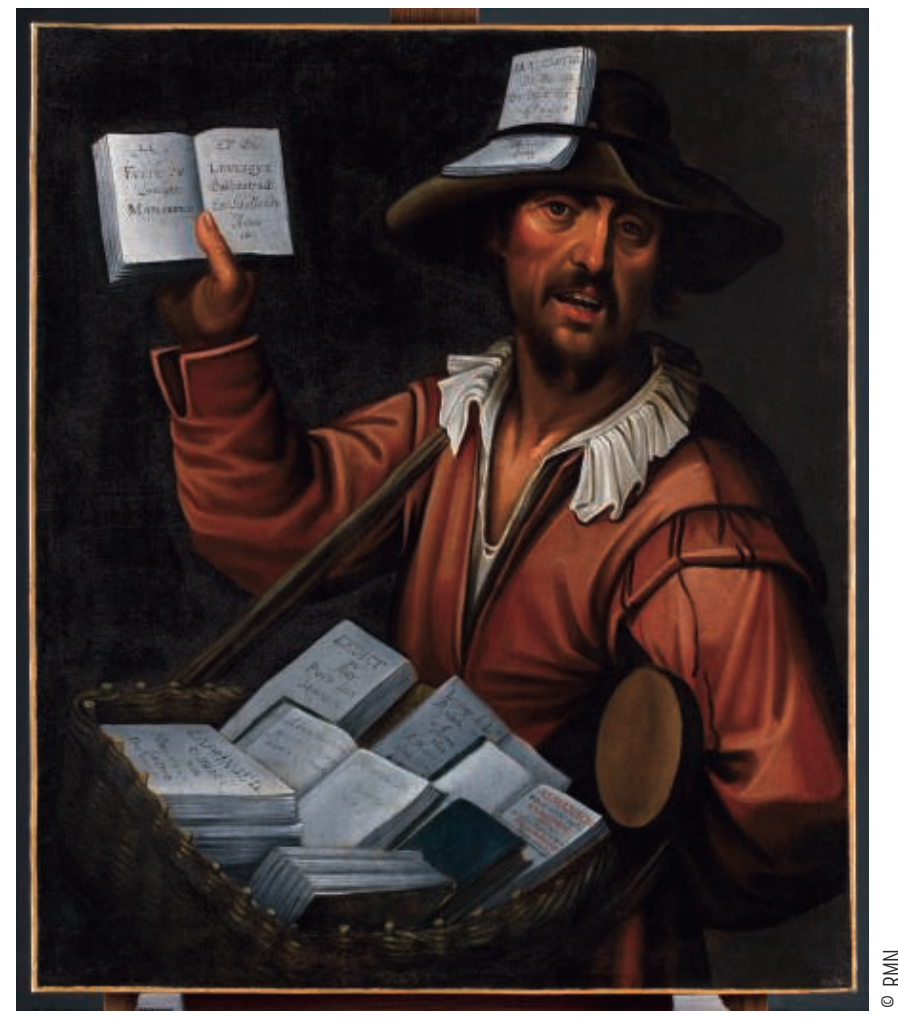

\section{Évolution au cours des siècles}

Le Bâtiment des recettes est un savant dosage de recettes domestiques, de recettes artisanales et de tours de charlatans. Dans un univers où l'autoconsommation est importante, il est à la fois un ouvrage utile et une source de divertissement, qui a su longtemps capter une large clientèle (Figure 7). Ce recueil technique est rédigé au Xvie siècle en langue vulgaire, et destiné à un marché local, contrairement à la littérature scientifique, rédigée en latin et destinée à une élite internationale. Son but n'est pas celui de la diffusion d’un savoir théorique, mais la transmission de savoirs pratiques ponctuels issus d'un constat dans l'expérience de l'échec et de la réussite à travers la répétition de gestes quotidiens. Ce type de littérature communique « des savoirs d'action, opératoires, prescriptifs, où priment le verbe et le geste, et qui postulent l'autonomie et la capacité participative de l'usager » (Hilaire-Perez \& Thébaud-Sorger 2006).

La magie fait intimement partie du Bâtiment des recettes, et de l'univers de la Renaissance où sciences occultes, sciences et techniques en général, ne peuvent être dissociées. La Renaissance occidentale ne conçoit pas une seule science homogène composée de plusieurs spécialités, mais des connaissances, des pratiques scientifiques qui s'appliquent dans un monde où l'on ne peut séparer le sacré du profane (Brioist 2002). Antoine Furetière, dans son Dictionnaire universel ${ }^{18}$, décrit la « magie naturelle » comme une science, mais une science du paradoxal, de l'étrange, de l'extraordinaire, car elle recherche l'incompréhensible : « science qui apprend à faire des choses surprenantes \& merveilleuses ». 


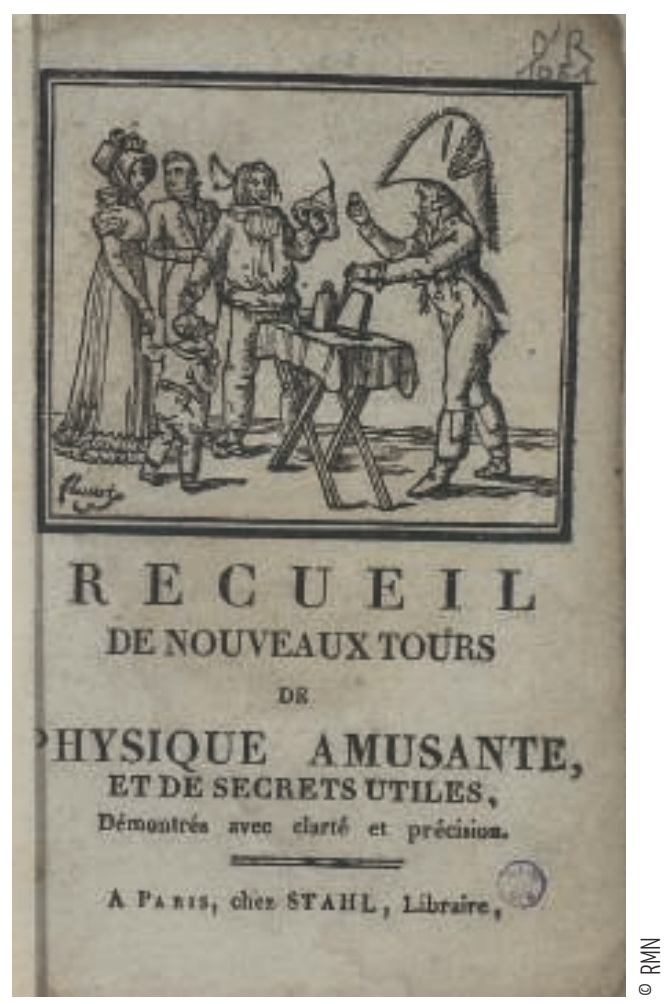

Jean Baptiste Porta, dit-il, « a éscrit de la Magie naturelle, des secrets pour faire des choses qui sont produites extraordinairement par des causes naturelles ».

La magie s'intègre parfaitement dans le recueil de recettes, au point que le lecteur ne puisse en délimiter les frontières. « Dans les sociétés où on ne fait pas de distinction fondamentale entre nature et surnature, on ne peut pas non plus distinguer la technique de la magie ». Cette réflexion de François Sigaut (Sigaut 2004) convient parfaitement aux pratiques décrites plus haut, relatives au détournement de l'usage des lampes à huile.

Le contenu de l'ouvrage offre une grande continuité du xvI au xix siècle, malgré une dégradation des éditions au xviII siècle (mise en page, coquilles). Il semble participer d'un monde parallèle à celui de la littérature savante, les XVII ${ }^{\mathrm{e}}$ XVIII et XIX ${ }^{e}$ siècles étant des périodes de bouleversements dans la pensée scientifique. L'absence fréquente de datation des éditions accentue ce décalage. À partir du xvil siècle, le projet éditorial n'est plus celui d'une construction, mais d'un recyclage : les quelques réactualisations se font en négatif par la suppression des paratextes, ou de certaines recettes.

Parallèlement, le monde de la physique expérimentale prend peu à peu le dessus. Le vocabulaire lui-même change de sens au cours du XVII siècle : la "physique », jusqu'alors synonyme de connaissance de la nature, ou de médecine, se transforme en une conception mathématique du monde. Les sciences et les arts et métiers deviennent à la mode. Les cabinets de physique et les livres de jeux scientifiques fleurissent. Les Leçons de physique expérimentale de l'abbé Nollet, éditées avec succès de 1745 à 1775, sont de véritables ouvrages de vulgarisation. Ils sont destinés à un public non averti d'hommes et de femmes issus d'un milieu aisé, tel ce Recueil de nouveaux tours de physique amusante, et de secrets utiles (Paris, s. d., Xville s.), paraissent aux xvIII et au xixe siècle, mêlant spectacle, plaisir, et utilité

(MuCEM). correspondant certainement à cette élite sociale qui a composé une partie du lectorat du Bâtiment des recettes aux siècles précédents. Ils offrent, explique Nollet, « un cours de physique expérimentale, et non pas un cours d'expériences».

La dégradation des éditions de colportage au XVIII ${ }^{e}$ siècle correspond probablement à une transformation et une dégradation de son lectorat : un décalage sociologique (Andries 1998) de plus en plus important apparaît en effet entre l'ouvrage du Xvi siècle figé par l'édition, et l'évolution des mentalités, entre l'élite sociale urbaine du xvi siècle à laquelle étaient destinées les recettes raffinées de parfums et de confitures, et le public alphabétisé de plus en plus large du XviII ${ }^{e}$ siècle.

Cependant, l'édition de colportage se met aussi au goût du jour : la Bibliothèque bleue, comme ses lecteurs, est elle aussi influencée, indirectement, par les Lumières : des livres de jeux et de prestidigitation qui revendiquent également un but éducatif paraissent à cette époque. « Nous avons... la prétention de plaire à ceux qui aiment à dépenser leurs loisirs à des distractions agréables et qui, dans des jeux en apparence frivoles, savent trouver une utile recette pour chasser l'ennui ou pour développer de plus en plus leur intelligence », est-il écrit dans un livre de Tours d'escamotage, de prestidigitation et d'adresse ${ }^{19}$. (Figure 8)

Par ailleurs, la magie perd sa dimension surnaturelle dans de nouveaux tours aux préoccupations toujours semblables, mais transformés en spectacles divertissants d'illusionnisme. Dans un livret intitulé Récréations amusantes, physiques et mathématiques, d’après les expériences du sieur MURELLE, escamoteur de nouveaux Tours, avec plusieurs autres belles expériences tirées de différents auteurs ${ }^{20}$, daté de 1778 , le thème des chandelles utilisées 
pour créer dans le noir des illusions d'optique est repris. Le titre annonce d'emblée qu'il s'agit de « récréations » sans autre prétention que l'amusement et le divertissement, avec cependant toujours le même vocabulaire : "Secret" pour faire paroître une compagnie en figure de bronze » (par un procédé chimique mêlant sel ordinaire, sucre blanc, esprit-devin et filasse) ${ }^{21}$. L'étrange et l'extraordinaire sont toujours là, mais le surnaturel s'évanouit au profit d'un bon tour utilisant des réactions chimiques ou bien la naïveté du lecteur. Le monde du quotidien des recueils de secrets, où se mêlent sciences, religion et magie existe cependant toujours au XVIII ${ }^{e}$ siècle, et pour longtemps encore. Mais il est alors coupé de la littérature "officielle». Le Bâtiment des recettes est même censuré au début du xvIII siècle par le Bureau de la Librairie. Cependant, cette censure n'empêche pas de nouvelles rééditions, mais celles-ci perdent de plus en plus en qualité et s'adressent à un lectorat de plus en plus large. (Figure 9)

Cette littérature déclassée aux yeux des élites, est toujours lue et entendue par le " grand public », ou public de non spécialistes, même si les différents contextes sociaux et historiques demandent quelques nuances. Robert Halleux et Carmelia Opsomer mentionnent la réflexion du folkloriste belge Albert Marinus au sujet des médecines dites populaires : « qu'elles soient des survivances de conceptions anciennes abandonnées par

Figure 9

On trouve dans cette édition de 1828 du Bâtiment des recettes, une copie grossière du frontispice du plus célèbre livre de secrets et de magie, les Admirables secrets d'Albert le Grand (Lyon, 1791) : un mage vêtu à l'orientale décrypte un grimoire posé sur une sorte d'autel, dans un cadre architectural théâtral. Cette allusion qui n'est accompagnée d'aucune transformation du texte, apporte une note sulfureuse attrayante, destinée à attirer un public friand de merveilleux (MuCEM).

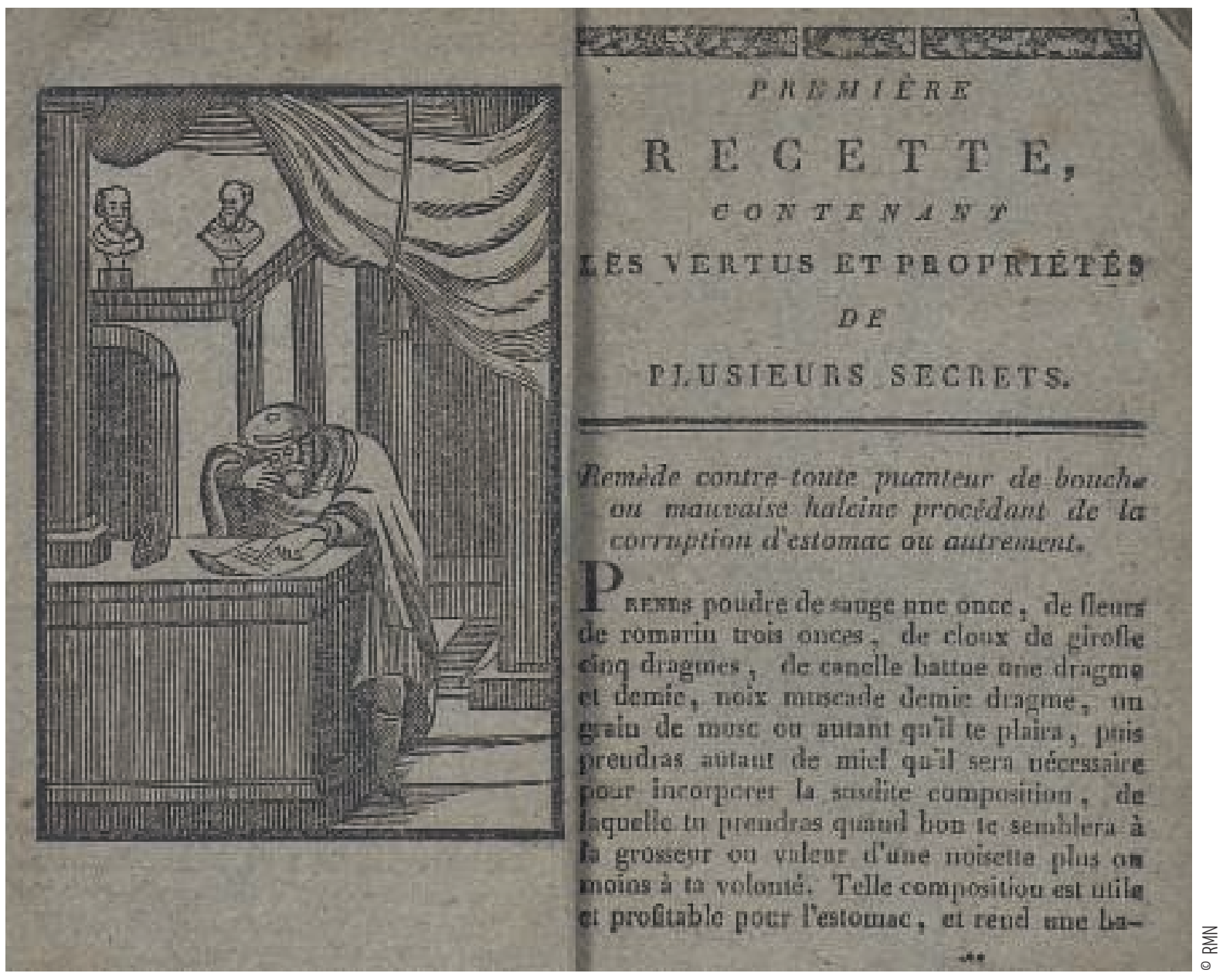


la science actuelle, c'est possible, mais elles sont en harmonie avec ceux qui en usent et y croient. Elles vivent donc » (Halleux \& Opsomer 2005). Ainsi, le Bâtiment des recettes peut être considéré comme un ouvrage de science populaire si l'on définit celle-ci comme une des formes de la pensée commune (Desile 2000). Dans le nouveau contexte du xviII siècle, cette forme littéraire qu'est le recueil de recettes ouvre elle aussi ses lecteurs à une méthode expérimentale, et les transforme en usagers autonomes.

L'humour et le divertissement qui émaillent l'ouvrage, ont leur part d'importance. Ils sont apportés par les recettes de bateleurs ou de charlatans. C'est le mode d'expression d'une littérature de vulgarisation dont on attend un plaisir de lecture. Ils donnent à l'ouvrage une dimension très sociable de "plaisir et joyeuseté ».

En faisant se côtoyer recettes pratiques, pratiques occultes, facéties et bons tours, ils mélangent différents niveaux d'écriture et de réception des messages. Ils contribuent ainsi à une distanciation face au texte imprimé, à une autonomie du lecteur expérimentateur.

Ils offrent également un certain goût de liberté face à l'ordre établi : les quelques recettes, peu nombreuses, pour se désenivrer, ou bien pour apprendre à « chaparder », transgressent l'interdit. Ce rire, issu de la culture populaire médiévale et renaissante, dit Mikhaill Bakhtine (1970 : 98), est en effet « comme une victoire sur la terreur mystique (« terreur divine ») et la peur qu'inspiraient les forces de la nature, mais avant tout comme une victoire sur la peur morale qui enchaînait, accablait, obscurcissait la conscience de l'homme, la peur de tout ce qui était sacré et interdit».

\section{$\&$}

Ainsi est amorcé sur le long terme un processus d'observation, de scepticisme, et de laïcisation du savoir-faire. Marcel Mauss (1902-1903) décrit bien ce processus. « Pour nous, écrit-il, les techniques sont comme des germes qui ont fructifié sur le terrain de la magie; mais elles ont dépossédé celle-ci. Elles se sont progressivement dépouillées de tout ce qu'elles lui avaient emprunté de mystique ; les procédés qui en subsistent ont, de plus en plus, changé de valeur ; on leur attribuait autrefois une vertu mystique, ils n'ont plus qu'une action mécanique; c'est ainsi que l'on voit de nos jours le massage médical sortir des passes du rebouteux ».

On peut alors considérer qu'un ouvrage tel que le Bâtiment des recettes, dont le but initial est la transmission d'un savoir, a sa place dans l'évolution générale des mentalités. En effet, parallèlement au déroulement de la révolution scientifique dans les milieux savants, la forme littéraire qu'est le recueil de recettes offre elle aussi une place importante à l'approche expérimentale des phénomènes : elle fait des ses lecteurs des expérimentateurs.

D'autre part, la structure du Bâtiment des recettes contribue à sa manière à une démystification du texte écrit et à une autonomie du lecteur expérimentateur, au travers de juxtapositions de recettes dont les contenus et les niveaux et de lecture différent.

La forme d'édition enfin, médiocre et bon marché, associée la forme de diffusion par colportage, lui confèrent une fonction économique, mais aussi culturelle, car elles contribuent à l'élargissement du public du livre, d'abord auprès d'une bourgeoisie urbaine, puis auprès d'une clientèle beaucoup plus large. Ce genre de littérature a donc probablement sa part d'importance dans le cheminement de l'ensemble de la société des Temps modernes vers ce que l'on pourrait appeler l'esprit critique. 


\section{NOTES}

En ouverture: Une étude de colporteur de Jean-Antoine Watteau, 1684-1721, Bayonne, musée Bonnat. (Cl. RMN)

1. Des formes très semblables existent dans d'autres pays d'Europe, comme les « chapbooks» anglais, ou la littérature de « cordel » en Espagne et au Brésil.

2. Selon Alain Viala, mentionné par JeanDominique Mellot (2009 : 43).

3. L'inventaire après décès du fonds de Jacques Oudot en 1722 répertorie 40000 volumes prêts à être livrés et 2576 rames de feuilles imposées, de quoi composer plus de 360000 livrets in- $8^{\circ}$ de 48 pages (cf. Mandrou, 1985).

4. Les mots « secret » et « recette » sont utilisés indifféremment durant tout le xvi siècle, prêtant éventuellement une dimension occulte à certaines recettes. Ensuite, ces deux mots peuvent avoir des significations différentes en fonction surtout du contexte, de l'époque et de leur identification à un certain type de savoir. Mais, le mot « secret » est longtemps conservé dans la littérature de colportage, comme un simple synonyme du mot « recette».

5. 58 éditions identifiées.

6. «Beau secret pour voir bien cler la nuyct... soit pour escripre ou estudier, ou faire quelque ouvraige de lyee comme taille Eaultre cas » $\left(35 \mathrm{r}^{\circ}\right)$.

7. Paris, B. Ste Geneviève en ligne sur Gallica : http:// www.archive.org/details/8T1540INV4101RES. Consulté en décembre 2012.

8. F. $42 \mathrm{r}^{\circ}$.

9. G. Della Porta, La Magie naturelle.

10. G. Della Porta, La Magie naturelle, f. $102 \mathrm{v}^{\circ}-103 \mathrm{r}^{\circ}$

11. G. Della Porta, La Magie naturelle, L. II, ch. 17, f. 131r ${ }^{\circ}$, "Des mesches de lampes ou chandelles, \& des illusions d'icelles, \& comme on pourra faire que les hommes seront veuz avoir testes de chevaux, ou d'autres animaux ».

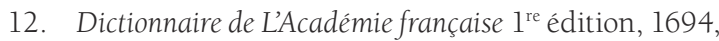
article « Magie ».

13. G. Della Porta, La Magie naturelle, L. II, ch. XV.

14. Dictionnaire de l'Académie française, 1694, et 1762 pour la deuxième assertion.

15. Est-ce une allusion à la doctrine de la vertu curative du rire héritée d'Hippocrate, très prisée à la faculté de médecine de Montpellier au XvI ${ }^{\mathrm{e}}$ siècle, que mentionne Mikhaill Bakhtine?

16. Les recettes du Plaisant jardin sont étudiées à partir de l'édition de Philippe Aline, Rouen, 1606, en ligne.

17. « liez... au col d'une anguille vive un petit sac de rongneures de vos ongles...»

18. Antoine Furetière, Dictionnaire universel, 1690, article Magie.

19. L'ancienne et la nouvelle collection des tours d'escamotage de prestidigitation et d'adresse... mis à la portée de tous, Paris, s.d. (MuCEM, Musée des Civilisations Européennes et Méditerranéennes, cote 1R 506).

20. MuCEM, cote O1R 743, p. 6, 7 .

21. Dans le Plaisant jardin, la recette «A faire que les gens ayent des testes comme de gens morts » (p. 112), prescrit : " prens de la cire vierge \& mets en fiente de cheval par neuf jours $\&$ neuf nuicts, $\&$ tu trouveras avec ladite cire petits vers, lesquels pilleras avec la dite cire \& en feras une chandelle, laquelle allumera [sic] là où il n'y aura autre lumière ».

\section{RÉEERERCES}

Andries, L. 1998 Les livres de secrets dans la littérature de colportage. In Curiosité et libido sciendi de la Renaissance aux Lumières. Fontenay-aux Roses : ENS éditions.

Bakhtine, M. 1970 L'Euvre de François Rabelais et la culture populaire au Moyen Âge et sous la Renaissance, trad. du russe par André Robel. Paris : Gallimard.

Barbier, F. 2012 Histoire du livre en Occident. Paris : Armand Colin.

Bollème, G. 1971 La Bibliothèque bleue. La littérature populaire en France du XVI e au XIXe siècle. Paris : Julliard.

Bollème, G. \& Andries, L. 2003 La Bibliothèque bleue. Littérature de colportage. Paris : Robert Laffont (Bouquins).

Brioist, P. 2002 Les savoirs scientifiques à la Renaissance. Revue d'histoire moderne et contemporaine 5/2002 (494bis), supplément : 52-80.

Chartier, R. 1983 Stratégies éditoriales et lectures populaires, 1530-1660. In R. Chartier \& H.-J. Martin (dir.) Histoire de lédition française, Tome I, Le livre conquérant. Du Moyen âge au milieu du xvII siècle. Paris : Promodis.

Deblock, G. (à paraître) Le «Bâtiment des recettes », un livre de secrets réédité du XVI au XIX siècle, mémoire de l'EPHE dirigé par Frédéric Barbier. 
Delcourt, Th. \& Parinet, I. (dir.) 2000 La Bibliothèque bleue et les littératures de colportage, actes du colloque organisé par la BMVR de Troyes en collaboration avec l'École Nationale des Chartes, à Troyes, 12-13 novembre 1999. Paris : École des Chartes ; Troyes : La Maison du Boulanger.

Della Porta, G. 1591 La Magie naturelle... qui est les secrets et miracles de nature. Lyon : Benoît Rigaud [1ère éd., Magia Naturalis, Naples : 1558].

Desile, P. 2000 Généalogie de la lumière. Paris : L'Harmattan.

Dictionnaire de l'Académie française 1694. Paris ; Chez la Veuve de Jean-Baptiste Coignard, Imprimeur ordinaire du Roy, \& de l'Académie Françoise

Dictionnaire de l'Académie française 1762 (4e édition). Paris : Chez la Vve B. Brunet.

Eamon, W. 1994 Science and the Secrets of Nature. Books of Secrets in Medieval and Early Modern Culture. Princeton : Princeton University Press.

- 2011 How to Read a Book of Secrets. In E. Leong et A. Rankin (eds) Secrets and Knowledge, in Medicine and Science, 1500-1800. Farnham : Ashgate.

Fontaine, L. 1993 Histoire du colportage en Europe, XV $v^{e}$-XIXe siècle. Paris : Albin Michel.

Furetière, A. 1690 Dictionnaire universel contenant généralement tous les mots françois, tant vieux que modernes, et les termes de toutes les sciences et des arts [édition posthume]. La Haye et Rotterdam : A. et R. Leers.

Halleux, R. \& Opsomer, C. 2005 L’Insaisissable médecine populaire. In P. Nobel (dir.) La Transmission des savoirs au Moyen âge et à la Renaissance. Besançon, Presses universitaires de Franche Comté.

Halleux, R. 2009 Le Savoir de la main, savants et artisans dans l'Europe pré-industrielle. Paris : Armand Colin.

Hilaire-Perez, L. \& Thébaud-Sorger, M. 2006 Les Techniques dans l'espace public. Publicité des inventions et littérature d'usage au xviII siècle (France, Angleterre). Revue de synthèse t. 127, $5^{e}$ série (2) : 393-428.

Mandrou, R. 1974 Introduction à la France moderne (1500-1640). Essai de psychologie historique. Paris : Albin Michel (L'évolution de l'humanité), transcrit des Archives départementales du Nord, B, 1817, inventaire impr., t. III.

— 1985 La Culture populaire aux XVII et XVIII siècles, Paris, Imago.

Martin, H.-J. 1975 Culture écrite et culture orale, culture savante et culture populaire dans la France d'ancien régime. Journal des savants, juillet-décembre (3) : 225-282.

Mauss, M. 1902-1903 Esquisse d'une théorie générale de la magie in Sociologie et anthropologie. Paris : P.U.F. (1968 $4^{e}$ édition).

- 1934 Les Techniques du corps. Communication présentée à la Société de psychologie le 17 mai 1934 in Sociologie et anthropologie. Paris : P.U.F. (Quadrige) 2008.

Mellot, J.-D. 1989 Rouen et les "libraires forains" à la fin du XviI" siècle : la veuve Machuel et ses correspondants (1768-1773). Bibliothèque de l'école des chartes, t. 147 : 503-538.

— 2009 La Capitale et l'imprimé à l'apogée de l'absolutisme (1618-1723). Histoire et civilisation du livre (5) : 17-44.

Morin, A. 1974 Catalogue descriptif de la bibliothèque bleue de Troyes (almanachs exclus). Genève : Droz.

Nollet, J.-A. 1745-1775 Leçons de physique expérimentale. Paris : Guérin Frères.

Oudin, A. 1971 [1640] Curiosités françaises, ou recueil de plusieurs belles propriétés avec une infinité de proverbes et de quolibets. Genève : Slatkine.

Sauvy, A. 1984 Le Livre aux champs. In R. Chartier \& H.-J. Martin (dir.) Histoire de l'édition française, tome II, Le livre triomphant, 1660-1830. Paris: Promodis.

Sigaut, F. 2010 La Formule de Mauss (nouvelle édition). Techniques \& Culture 54-55 Cultures matérielles, vol. 1 Anthologie raisonnée de Techniques \& Culture : 354-367 ; 2002 (première édition). Efficacité technique, efficacité sociale, Techniques et culture 40 [en ligne http://tc.revues.org/5007 consulté le 9 mars 2013].

— 2004 Les Techniques dans la pensée narrative. Mythes. L'origine des manières de faire, Techniques et culture 43 44 [en ligne http://tc.revues.org/1241 consulté le 9 mars 2013].

Smith, Pamela H. 2011 What is a Secret? Secrets and Craft Knowledge in Early Modern Europe. In E. Leong et A. Rankin (dir.) Secrets and Knowledge in Medicine and Science, 1500-1800. Farnham : Ashgate.

Spufford, M. 1984 The Great Reclothing of Rural England. Petty Chapmen and their Wares in the Seventeenth Century. Londres : Hambledon Press. 


\section{RÉSUMÉ}

Astuces, farces, magie. Les recettes de divertissements du "Bâtiment des recettes " (XVI ${ }^{e}$-XIX ${ }^{e}$ siècles). Le Bâtiment des recettes est un recueil de secrets techniques. Constitué au $\mathrm{XvI}^{e}$ siècle en langue vulgaire, il est destiné à un marché local. Son texte, édité durant presque quatre siècles pour une population diverse et nombreuse, offre une grande continuité du Xvi au XIX siècle. L'humour et le divertissement qui émaillent l'ouvrage sont apportés par les recettes de bateleurs ou de charlatans, et ont leur part d'importance parmi les multiples préoccupations domestiques et artisanales. La juxtaposition de recettes pratiques, de magie, de facéties et de bons tours permet en effet de mélanger différents niveaux d'écriture et de réception des messages. Cela donne une dimension très sociable de " plaisir et joyeuseté », contribue à une distanciation par rapport au texte imprimé, et apporte un certain goût de liberté ou d'autonomie. Au XviII siècle, un décalage sociologique de plus en plus grand apparaît entre l'ouvrage du Xvi siècle figé par l'édition, et l'évolution des mentalités. Cependant, cette littérature déclassée aux yeux des élites est toujours éditée et vendue : elle représente encore une des formes de la pensée commune. On peut donc considérer qu'un ouvrage tel que le Bâtiment des recettes, édité durant quatre siècles, a sa place dans l'évolution générale des mentalités. Parallèlement au déroulement de la révolution scientifique dans les milieux savants, il offre lui aussi une place importante à l'approche expérimentale des phénomènes, et contribue à sa manière à une démystification du texte écrit. Ce genre de littérature, largement répandue, a probablement sa part d'importance dans le cheminement de l'ensemble de la société des Temps modernes vers ce que l'on pourrait appeler l'esprit critique.

\section{ABSTRACT}

Tips, tricks and magic. The Bâtiment des recettes is a collection of technical secrets. Compiled in the $16^{\text {th }}$ century in common language, it is intended for a local market. Its text, published during almost four centuries for a diverse, numerous population, presents great continuity from the $16^{\text {th }}$ to the $19^{\text {th }}$ century. The humour and entertainment that pepper the book come from tumblers and charlatans'recipes and are quite important among the many domestic and craft concerns. In fact, the combination of practical recipes, magic, tricks and pranks makes it possible to mix different registers of language and comprehension. This conveys a very sociable aspect of pleasure and merriment, creates a distance with the printed text and brings about a certain taste of freedom and autonomy. In the $18^{\text {th }}$ century, a growing sociological gap appears between the $16^{\text {th }}$ century book, rigidly fixed by the edition, and the evolution of mentalities. However, this literature, downgraded in the elite's opinion, is still published and sold : it remains one of the forms of common thought. We can therefore consider that a book such as the Bâtiment des recettes, edited throughout four centuries, has its place in the general evolution of mentalities. Parallel to the course of scientific revolution in learned milieus, it too offers an important place in the experimental approach to phenomena and contributes, in its own way, to a demystification of the written text. This widespread type of literature is probably quite important in the progression of the whole society of Modern Times towards what could be called the critical mind.

\section{MOTS CLÉS}

Littérature technique, littérature de colportage, magie, divertissement, illusionnisme, trucs et astuces, histoire des mentalités, histoire du livre, diffusion, $\mathrm{XvI}^{\mathrm{e}}$-XIX ${ }^{\mathrm{e}}$ siècles

\section{KEYWORDS}

Technical literature, literature about peddling, magic, entertainment, conjuring, tricks and clever devices, historie of mentalities, historie of the book, diffusion, $16^{\text {th }}-19^{\text {th }}$ century 